\title{
Overexpression of Aiolos in Nalm-6 acute lymphoblastic leukaemia cells reduces apoptosis by suppressing phosphatase and tensin homologue deleted on chromosome 10 and activating the phosphatidylinositol-3-kinase/Akt signalling pathway
}

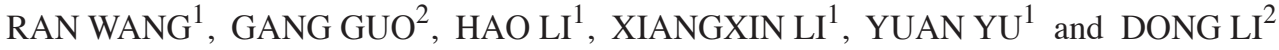 \\ ${ }^{1}$ Department of Hematology; ${ }^{2}$ Cryomedicine Laboratory, Qilu Hospital, \\ Shandong University, Ji'nan, Shandong 250012, P.R. China
}

Received March 22, 2014; Accepted December 12, 2014

DOI: $10.3892 / \mathrm{mmr} .2015 .3214$

\begin{abstract}
The aim of the present study was to elucidate the molecular mechanism of Aiolos in the regulation of B-cell leukaemia. A lentiviral system was used for overexpression of the Aiolos gene in Nalm- 6 cells to determine the effects of Aiolos on proliferation, apoptosis and the cell cycle. The expression and activation of phosphatase and tensin homolog deleted on chromosome ten (PTEN) and Akt were also investigated. Upregulation of Aiolos inhibited cell growth and arrested an increased number of Nalm- 6 cells at the G0/G1 phase. The apoptotic cell quantities were also significantly lower in the Aiolos-transfected Nalm-6 cells. In addition, Aiolos overexpression downregulated PTEN, but increased the expression and phosphorylation of Akt in the Nalm- 6 cells. The Akt inhibitor, Akti-1/2, reduced the percentage of viable Aiolos-overexpressed Nalm-6 cells, however, it had no effect on cell cycle arrest or proliferation. Aiolos upregulation in the Nalm- 6 cells inhibited cell proliferation, suppressed apoptosis and arrested the cell cycle at the G0/G1 phase. Aiolos improved the survival of Nalm- 6 cells via PTEN- and Akt-dependent processes.
\end{abstract}

\section{Introduction}

Acute lymphoblastic leukaemia (ALL) is a malignant disorder of lymphoid progenitor cells. ALL is the most common type of childhood malignancy and accounts for almost $20 \%$ of cases of acute leukaemia in adults. B-cell precursor ALL (BCP-ALL)

Correspondence to: Mr. Dong Li, Cryomedicine Laboratory, Qilu Hospital, Shandong University, 107 Wenhuaxilu Road, Ji'nan, Shandong 250012, P.R. China

E-mail: lidong73@sdu.edu.cn

Key words: Aiolos, B-cell acute lymphoblastic leukaemia, apoptosis, phosphatase and tensin homologue deleted on chromosome ten, phosphatidylinositol-3-kinase/Akt signalling pathway is the most common type of childhood ALL (1). Survival rates have markedly improved with combination chemotherapy and treatment intensification; however, the occurrence of minimal residual disease has prompted further examination of the molecular mechanisms underlying ALL (2).

BCP-ALL is a malignancy characterised by the progressive accumulation of immature clonal B-cell precursors in the bone marrow. Numerous transcriptional regulators have critical functions in this malignant process and may regulate the expression of genes, whose products affect the fate and function of lymphoid cells (3). The Ikaros family of proteins is an example of these factors (4), which encode zinc-finger DNA-binding proteins (5-7). Aiolos, the second Ikaros family member identified, is of particular interest in the investigation of BCP-ALL (6). Aiolos is an important regulator of B-cell differentiation, proliferation and maturation to an effector state. Disruption of Aiolos increases the number of pre-B and immature $B$ cells, and significantly reduces the number of circulating B cells (8). In addition, an Aiolos null mutation in mice causes B-cell hyperproliferation, elevates serum antibody levels, promotes auto-antibody formation and triggers lymphoma development, thereby demonstrating tumour suppressor function (8). The deregulated expression of Aiolos has been associated with adult B-cell ALL and chronic lymphocytic leukaemia (CLL) in human patients (9-11) and aberrant expression levels of Aiolos levels been reported in cases of lymphoma (12).

However, the molecular mechanisms through which Aiolos exerts its growth inhibitory effect remain to be elucidated. The Aiolos gene is crucial in lymphoid development and apoptosis; therefore, the function of Aiolos in childhood BCP-ALL and the downstream regulatory mechanisms of Aiolos require investigation. In the present study, a lentiviral system was used to achieve stable overexpression of the Aiolos gene in the Nalm-6 BCP-ALL cell line. Subsequently, the effects of Aiolos upregulation on the proliferation, apoptosis and cell cycle distribution of Nalm-6 cells in vitro was examined. Further experiments indicated that the Akt, protein kinase, signalling pathways were regulated by loss of phosphatase and tensin homologue deleted on chromosome ten (PTEN) caused by overexpression of Aiolos. 


\section{Materials and methods}

Recombinant lentiviral vector production. Aiolos cDNA (NM_012481; YRgene, Changsha, China) was cloned into a pWPT-PURO-green fluorescent protein (GFP) plasmid (Shanghai Telebio Biomedical Co. Ltd., Shanghai, China), which was co-transfected with three packaging plasmids (pRsv-REV, pMD1g-pRRE and pMD2G; Shanghai Telebio Biomedical Co. Ltd.) into human embryonic kidney (HEK)293T cells (Shanghai Telebio Biomedical Co. Ltd.). The HEK293T cells were plated at $7 \times 10^{5}$ on six-well plates (Corning Costar; Corning, Inc., New York, NY, USA) in $2 \mathrm{ml}$ of DMEM (Invitrogen Life Technologies, Carlsbad, CA, USA) with $10 \%$ foetal bovine serum (FBS; Gibco-BRL, Grand Island, NY, USA) and no antibiotics, and incubated at $37^{\circ} \mathrm{C}$ with $5 \% \mathrm{CO}_{2}$, overnight. Subsequently, $10 \mu \mathrm{g}$ pRsv-REV, $15 \mu \mathrm{g}$ pMDlg-pRRE and $7.5 \mu \mathrm{g}$ pMD2G packaging plasmids diluted in $1600 \mu 1$ of Opti-MEM-I (Invitrogen Life Technologies) were combined, and $20 \mu \mathrm{g}$ pWPT-PURO-GFP-Aiolos lentiviral DNA diluted in $200 \mu \mathrm{l}$ of Opti-MEM-I was added to the tube. Finally $200 \mu \mathrm{l} \mathrm{CaCl}{ }_{2}$ $(2.5 \mathrm{~mol} / \mathrm{l})$ was added to the tube, directly into the media to bring the final reaction volume to $2000 \mu 1$. The tube was agitated and then incubated at room temperature for $30 \mathrm{~min}$. The reaction mixture $(300 \mu \mathrm{l})$ was added to each well of the HEK 293T cells plated the previous day, and incubated at $37^{\circ} \mathrm{C}, 5 \% \mathrm{CO}_{2}$, overnight. The following day the media was aspirated and replaced with $2 \mathrm{ml}$ fresh DMEM containing 10\% FBS and 1\% PenStrep (Invitrogen Life Technologies), and incubated overnight. The virions, released into the media, were collected 48 and $72 \mathrm{~h}$ after transfection. To concentrate the viral particles, the media were ultracentrifuged twice at $106,000 \times \mathrm{g}(1.5 \mathrm{~h} /$ round $)$ and the final pellet was dissolved in RPMI 1640 (Invitrogen Life Technologies), 10\% FBS and $1 \%$ glutamine (Invitrogen Life Technologies). Lenti-GFP was constructed similarly, but without the Aiolos insert.

Cell culture and transfection. The BCP-ALL cell line Nalm-6 was purchased from the American Type Culture Collection. (Manassas, VA, USA) and cultured in standard RPMI 1640 medium (Gibco-BRL) containing 10\% foetal bovine serum (Gibco-BRL) and 1\% penicillin-streptomycin (Gibco-BRL) at $37^{\circ} \mathrm{C}$ in $5 \% \mathrm{CO}_{2}$. The Nalm- 6 cells were divided into the following three groups: Untransfected control (UT), Lenti-GFP group and Aiolos-transfected (Lenti-Aiolos) group. The viral concentrate was diluted in polybrene (5 $\mu \mathrm{g} / \mathrm{ml}$; Sigma-Aldrich, St. Louis, MO, USA) to infect the Nalm- 6 cells at a multiplicity of infection of 100. Successful transduction was confirmed by visualisation of enhanced GFP after 4 days using a fluorescence microscope (IX71; Olympus Corp., Tokyo, Japan) and Phenix micro-image analysis software version 2.2 (Phenix Optical Holding Stock Co., Ltd, Jiangxi, China). The cells were maintained and allowed to grow at $37^{\circ} \mathrm{C}$ for an additional 6 days, following which the expression levels of Aiolos were confirmed by reverse transcription-quantitative polymerase chain reaction (RT-qPCR) and western blot analysis. The virus-infected cells were selected with $8 \mu \mathrm{g} / \mathrm{ml}$ puromycin (Invitrogen Life Technologies, Carlsbad, CA, USA). The antibiotic-resistant clones were pooled and used for subsequent assays.
Treatment with Akt inhibitor. Akti-1/2, previously known as Akt-I-1/2, was purchased from Sigma-Aldrich and reconstituted in dimethyl sulfoxide (DMSO; Sigma-Aldrich) to a stock concentration of $50 \mathrm{mM}$. The final concentration of DMSO in the cultures was maintained at $0.1 \%$. For all the experiments, the cells $\left(2 \times 10^{5} / \mathrm{ml}\right)$ were treated with $0.5 \mu \mathrm{M}$ Akti- $1 / 2$ at $37^{\circ} \mathrm{C}$ for $48 \mathrm{~h}$ and treatment was continued for subsequent experiments. The control cells were treated with equal quantities of the solvent. All experiments were performed in triplicate.

Cell growth curve. The Nalm- 6 cells of the UT, Lenti-GFP and Lenti-Aiolos groups were seeded into 24 -well plates (Corning Costar) in triplicate at a density of $2 \times 10^{5}$ per well at $37^{\circ} \mathrm{C}$ seven days after transfection. The number of live cells was determined by trypan blue staining (Sigma-Aldrich) and was measured daily for 5 days using a haemocytometer (Jingmai Biotech., Ji'nan, China) under an inverted microscope (IX71; Olympus Corp., Tokyo, Japan).

Cell cycle analysis. For cell cycle analysis, a total of $1 \times 10^{6}$ cells were first fixed with $1 \mathrm{ml}$ ice-cold $70 \%$ ethanol and then incubated with RNase A at $37^{\circ} \mathrm{C}$ for $30 \mathrm{~min}$. The cells were stained with propidium iodide (PI; $50 \mu \mathrm{g} / \mathrm{ml}$ PI in $0.1 \%$ sodium citrate and $0.1 \%$ Nonidet P-40; Sigma-Aldrich) for $30 \mathrm{~min}$ at $4^{\circ} \mathrm{C}$. The cells were then analysed using a fluorescence-activated cell sorting (FACS)Calibur instrument equipped with Cell Quest software version 5.0 (BD Biosciences, Franklin Lakes, NJ, USA). The results are expressed as the percentage of the cells in each cell cycle phase and error bars indicate the standard deviation of the mean (SEM).

Detection of apoptosis. Annexin V/PI labelling was performed using the Vybrant Apoptosis Assay kit \#3 (Invitrogen Life Technologies), according to the manufacturer's instructions, followed by flow cytometry. The Nalm- 6 cells were scanned in fluorescein isothiocyanate (FL1-H), vs. PI (FL2-H) channels using a FACSCalibur instrument and Cell Quest software version 5.0.

Isolation of RNA and RT-qPCR analysis. Total RNA was extracted from each group of Nalm- 6 cells using TRIzol reagent (Invitrogen Life Technologies), according to the manufacturer's instructions. Purified total RNA $(1 \mu \mathrm{g})$ was reverse transcribed to cDNA using the Omniscript cDNA synthesis kit (Qiagen, Hamburg, Germany) using random primers. The relative gene expression levels were measured by qPCR using gene-specific primers and SYBR Green supermix (Promega Corp., Madison, WI, USA). $\beta$-actin was used as an endogenous control. The primers used are listed in Table I.

Western blot analysis. The proteins were extracted using radioimmunoprecipitation assay buffer containing $1 \mathrm{X}$ phosphate-buffered saline, $1 \%$ Nonidet P- $40,0.5 \%$ sodium dexoycholate, $0.1 \%$ SDS and protease inhibitor mixture tablet (Roche Diagnostics GmbH, Mannheim, Germany). At least $20 \mu \mathrm{g} /$ lane proteins were subjected to $12 \%$ SDS-PAGE and subsequently transferred to polyvinylidene fluoride hydrophobic membranes (EMD Millipore, Bedford, MA, USA). 
Table I. Primers used in reverse transcription-quantitative polymerase chain reaction.

\begin{tabular}{lll}
\hline Gene & Forward/reverse & \multicolumn{1}{c}{ Sequence } \\
\hline PTEN & Forward & Product (bp) \\
& Reverse & 5'-GTGGCGGAACTTGCAATCCT-'3 \\
Akt1 & Forward & 5'-CGGCTGAGGGAACTCAAAGT-'3 \\
& Reverse & 5'-ATGGACAGGGAGAGCAAACG-'3 \\
Akt2 & Forward & 5'-CTGGCCACAGCCTCTGATG-'3 \\
& Reverse & 5'-GGTGACAGACTGTGCCCTG-'3 \\
Akt3 & Forward & 5'-GGTACGCTGTCACCTAGCTC-'3 \\
& Reverse & 5'-TTGGTTCGAGAGAAGGCAAGT-'3 \\
GAPDH & Forward & 5 '-GTGTGCCACTTCATCCTTTGC-'3 \\
& Reverse & 5 '-ATAAATTGAGCCCGCAGCC-'3 \\
\hline
\end{tabular}

PTEN, phosphatase and tensin homolog deleted on chromosome ten.

Membranes were blocked with 5\% nonfat dry milk (Shifeng Biotech) in Tris-buffered saline containing $0.05 \%$ Tween-20 (TBST; Sigma-Aldrich) at $4^{\circ} \mathrm{C}$ for $2 \mathrm{~h}$. The membranes were rinsed with TBST and incubated with rabbit monoclonal Aiolos antibody (1/20;000; cat. 39408; Abcam, Cambridge, MA, USA), rabbit monoclonal PTEN antibody (1/500; cat. 32199; Abcam), rabbit monoclonal Akt (1/10,000; cat. 32505; Abcam), rabbit polyclonal phosphorylated-Akt antibody (Thr-308, 1/2,000; cat. 66134; Abcam) and rabbit monoclonal $\beta$-actin (1/20,000; cat. 79467; Abcam). Horseradish peroxidase-conjugated secondary antibodies (goat anti-rabbit immunoglobulin G H\&L-HRP; 1/10,000; cat. 16721; Abcam) and an Enhanced Chemiluminescence (ECL) Plus Immunoblotting Detection system (Beyotime, Shanghai, China) were used to detect specific binding, and images of the signals were captured using X-ray films (Kodak, Rochester, NY, USA) and WD-9413C Electrophoresis image analysis system (Liuyi, Beijing, China).

Statistical analysis. All data are presented as the mean \pm standard deviation and analysed via analysis of variance, which was followed by Student's t-test. $\mathrm{P}<0.05$ was considered to indicate a statistically significant difference. All statistical analyses were conducted using the SPSS 13.0 software program (SPSS Inc., Chicago, IL, USA).

\section{Results}

Aiolos overexpression by stable transfection in Nalm-6 cells. The Nalm- 6 cells were infected with the pWPT-PURO-GFP-Aiolos lentivirus vector, which was inserted with the entire Aiolos coding sequence, to induce the upregulation of Aiolos. As a control, Nalm- 6 cells were either infected with a lentivirus vector expressing GFP or were not infected. At 4 days after infection, the infection efficiency was determined based on the expression level of GFP using a fluorescence microscope. A substantial number of cells emitted bright green fluorescence, suggesting high infection efficiency (Fig. 1A-F). The cells were maintained in culture and grown for an additional 3 days. Subsequently, the mRNA and protein expression levels of Aiolos in the Nalm-6 cells of the three groups were determined by RT-qPCR and western blot assays. The RT-qPCR results demonstrated that the mRNA expression of Aiolos in the Nalm- 6 cells of the Lenti-Aiolos group was 1.5-fold higher compared with that in the Nalm- 6 cells of the UT group ( $\mathrm{P}<0.01$; Fig. $1 \mathrm{G})$. This result is consistent with the increase in the protein expression of Aiolos (Fig. 1H). No significant difference was observed in the distribution of expression of Aiolos between the Lenti-GFP group and UT group.

Overexpression of Aiolos inhibits the growth of Nalm-6 cells and leads to cell cycle arrest at the G0/G1 phase. The growth properties of the Lenti-Aiolos group were measured and compared with those of the control Lenti-GFP transfected group to determine whether overexpression of Aiolos affects the proliferation of Nalm-6 cells. As shown in Fig. 2A, the growth rate of the Lenti-Aiolos group was significantly lower compared with that of the Lenti-GFP or UT group. This result suggested that the upregulation of Aiolos may inhibit the growth of Nalm- 6 cells.

The resulting inhibition of the proliferation of Nalm- 6 cells infected with Lenti-Aiolos compared with control Nalm-6 cells may be, in part, caused by differences in cell cycle regulation. Thus, the cell cycles of Nalm- 6 cells in different groups were characterised by FACS analysis 9 days after transfection. No significant difference was detected between the Lenti-GFP and Nalm-6 cells (Fig. 2B; P>0.05). However, the percentage of Nalm- 6 cells in the G0/G1 phase increased between $72 \%$ (UT) and $84.5 \%$ (Lenti-Aiolos; $\mathrm{P}<0.01$ ), and the percentage of Nalm-6 cells in the S-phase cells decreased between $19 \%$ (UT) and 10.9\% (Lenti-Aiolos; $\mathrm{P}<0.01$ ). A significant difference in the percentage of Nalm- 6 cells in the G2/M phase was found between the Lenti-Aiolos group and the UT group (4.5. vs. 9\%, respectively; $\mathrm{P}<0.01$ ). These data indicated that Aiolos upregulation arrested an increased number of Nalm-6 cells at the G0/G1 phase, which possibly contributed to the growth inhibition observed.

Aiolos overexpression suppresses the apoptosis of Nalm-6 cells. An apoptosis assay kit was used to measure cell 

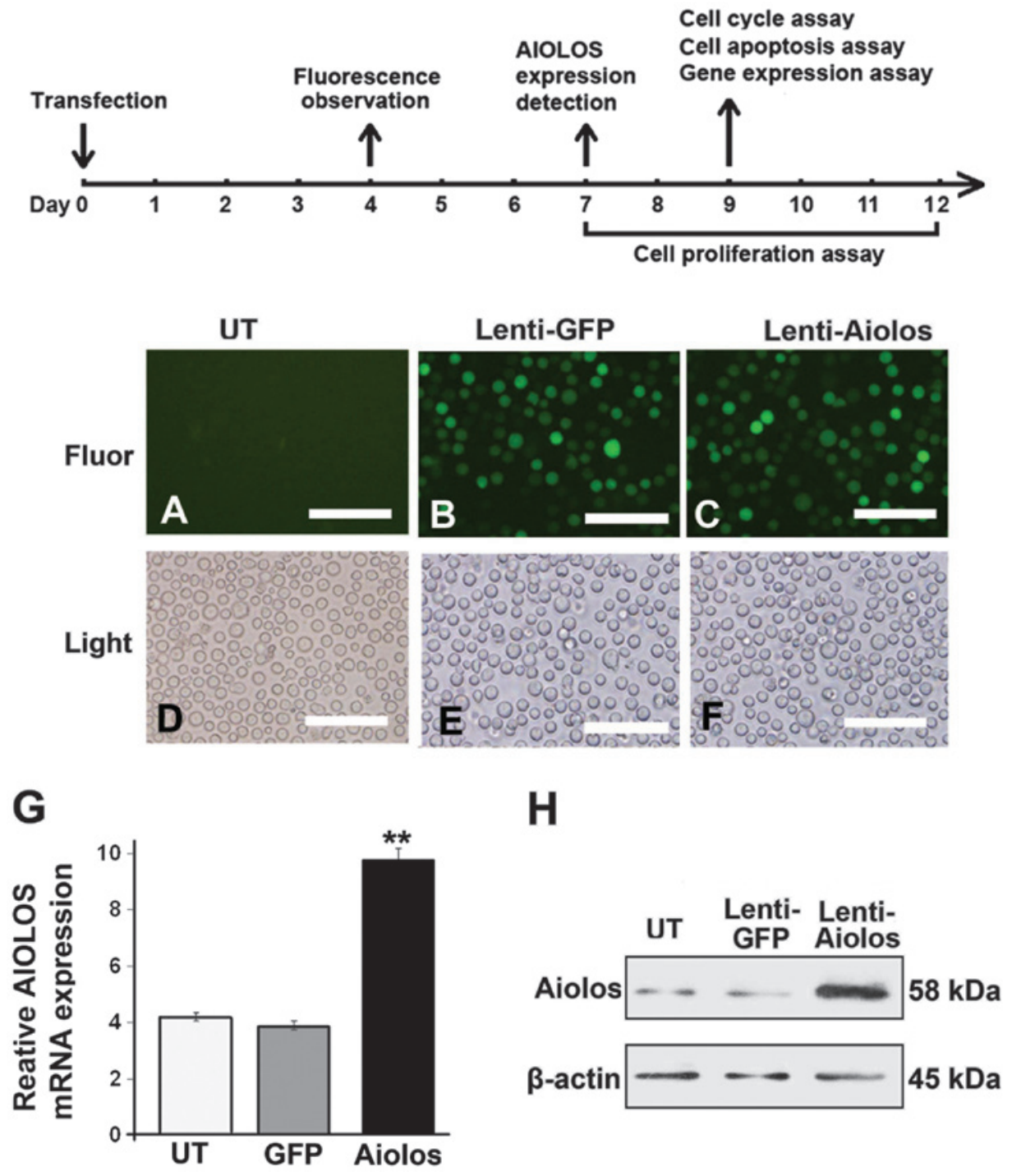

Figure 1. Timeline of the treatment of Nalk-6 cells involving the overexpression of Aiolos. (A-F) Lentiviral transduction efficiency in the Nalm-6 cells. Transduction efficiency was estimated 4 days after infection at a multiplicity of infection of 100. The expression of GFP was observed under (A, B and C) fluorescence or (D, E and F) light microscopy. Scale bar: $200 \mu \mathrm{m}$ (magnification, x200). (G) Reverse transcription-quantitative polymerase chain reaction of the relative transcription levels of Aiolos in the UT, Lenti-GFP, and Lenti-Aiolos groups. $\beta$-actin was used as an internal control. (H) Western blotting results revealed that protein expression of Aiolos was present in the Nalm- 6 cells of the three groups. $\beta$-actin was used as a loading control. Error bars indicate the standard deviation of the mean of three experiments. ${ }^{* *} \mathrm{P}<0.01$ Lenti-Aiolos group vs. Lenti-GFP group. GFP, green fluorescent protein; UT, untransfected control; Lenti-GFP, lentiviral vector control; Lenti-Aiolos, Aiolos-transfected; Fluor, fluorescence.

apoptosis 9 days after transfection to determine whether overexpression of Aiolos affects the apoptosis of Nalm-6 cells. As shown in Fig. 3, the total number of apoptotic cells was significantly lower in the Aiolos-transfected Nalm-6 cells (11\%) compared with the cells in the Lenti-GFP (16.5\%) or UT groups $(18.9 \%$; $\mathrm{P}<0.05)$. A minimal difference in the percentage of early apoptotic cells was found between the Aiolos-transfected and UT Nalm-6 cells (8.05, vs. $11.2 \%$; $\mathrm{P}>0.05)$, whereas a significant difference was observed in the percentage of late apoptotic cells between the cell groups (2.95, vs. $7.7 \%$; $\mathrm{P}<0.05)$. The obtained data suggested that overexpression of Aiolos may suppress apoptosis in Nalm-6 cells.

Upregulated Aiolos suppresses the expression of PTEN, but activates certain phosphatidylinositol-3-kinase (PI3K)/Akt signalling pathway-associated genes in Nalm-6 cells. Microarrays were used to analyse the gene expression of Nalm-6 cells transfected with Aiolos and to further examine the underlying mechanism responsible for the aforementioned changes in biological behaviour (data not shown). Among the deregulated genes, several key genes are of note. The expression levels of PTEN and certain Akt family genes were further detected using RT-qPCR and western blot analysis. The RT-qPCR results revealed that the expression level of PTEN in the Nalm- 6 cells was significantly lower in the Lenti-Aiolos group compared with the control group $(\mathrm{P}<0.05)$. In addition, upregulation of Akt1 $(\mathrm{P}<0.05)$ and Akt2 $(\mathrm{P}<0.01)$ was confirmed in the Lenti-Aiolos group. No significant change was observed in the expression of Akt3. Consistent with the RT-qPCR results, decreased expression levels of PTEN was observed by western blot analysis. The protein expression of Akt did not change considerably, whereas the levels of phosphorylated Akt were significantly 
A

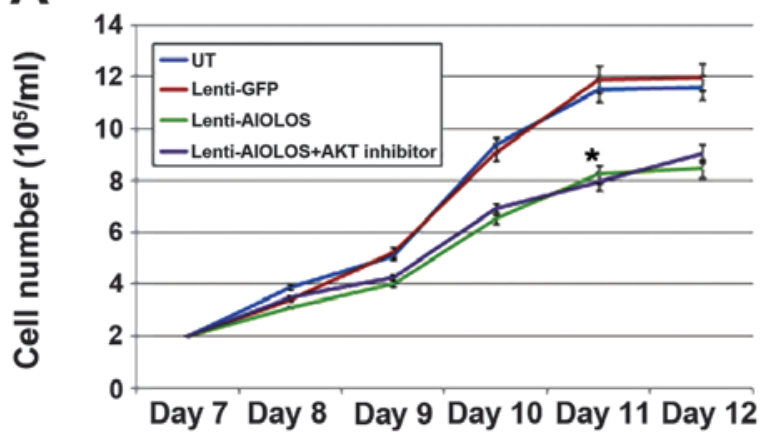

B

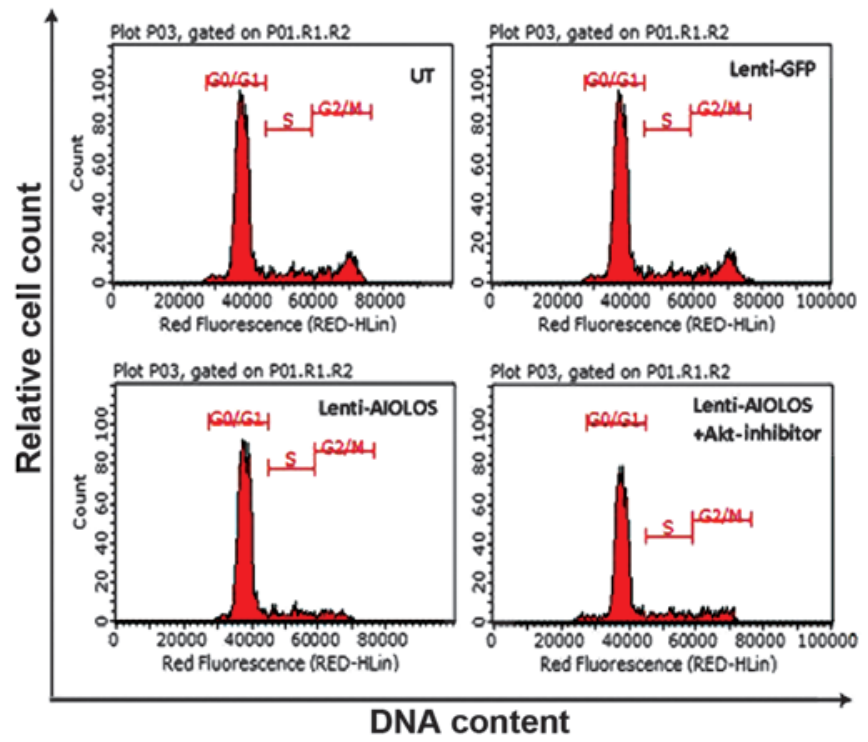

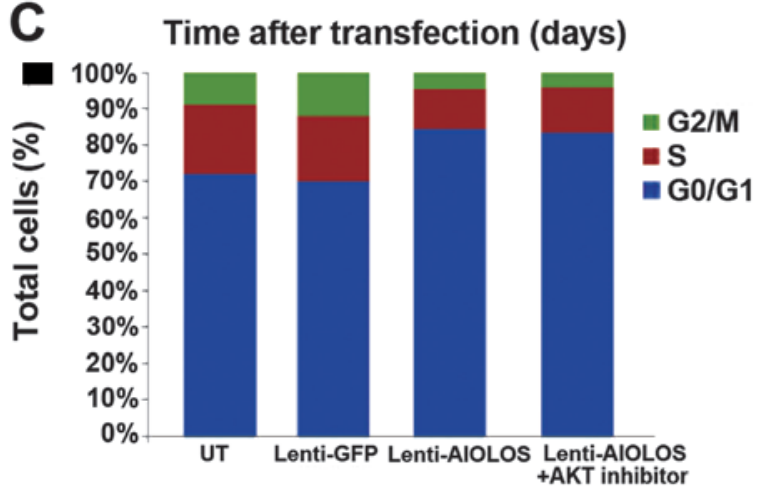

Figure 2. Effect of overexpression of Aiolos on the proliferation and cell cycle distribution of Nalm- 6 cells. The numbers of Nalm- 6 cells in the three groups were counted between day 7 and day 12 following transfection. Each time-point was counted in triplicate. (A) Proliferation of Nalm-6 cells in the Lenti-Aiolos group reduced by $16 \%$ on day 9 compared with that of the UT group cells ( $\mathrm{P}>0.05)$. The reduction peaked at $29 \%$ on day 11 ( $\mathrm{P}<0.05$ vs. UT group cells). The same proliferative capacity was observed in the Lenti-GFP group and the UT group (P>0.05 vs. UT group). Treatment with chemical inhibitors of Akt (Akti-1/2) did not improve the growth of the Nalm-6 cells in the Lenti-Aiolos group. (B and C) Flow cytometric analysis demonstrated that the proportions of cells in the G0/G1 phase increased and the proportion of cells in the G2/M and S phase decreased in the Nalm-6 cells of the Lenti-Aiolos group compared with those in the Lenti-GFP group ( $\mathrm{P}<0.01$ vs. Lenti-GFP group). Akti-1/2 did not affect the cell cycle of the Lenti-Aiolos group. Error bars indicate the standard deviation of the mean from three experiments). GFP, green fluorescent protein; UT, untransfected control; Lenti-GFP, lentiviral vector control; Lenti-Aiolos, Aiolos-transfected.

higher in the Lenti-Aiolos group compared with the control group (Fig. 4).

Akt inhibitors reverse Aiolos overexpression-induced apoptosis, but do not affect proliferation arrest. The cells were incubated with varying concentrations of the inhibitor, Akti-1/2, (0.1-10 $\mu \mathrm{M})$ for $48 \mathrm{~h}$ and cell viability was measured to verify whether the activation of Akt was principally responsible for the decreased apoptosis in the Aiolos-overexpressed Nalm- 6 cells. The results of the western blotting revealed that Akti-1/2, at a concentration of $5 \mu \mathrm{M}$, effectively inhibited Akt phosphorylation. In addition, Akti-1/2 effectively increased the percentage of apoptotic cells in the Aiolos-transfected Nalm-6 cells (Fig. 3). However, in the cell proliferation experiments, Akti-1/2 treatment did not improve the growth of the Nalm- 6 cells in the Lenti-Aiolos group (Fig. 2A). Similarly, Akti-1/2 did not affect the cell cycle of the Lenti-Aiolos group (Fig. 2B and C).

\section{Discussion}

In the present study, the Nalm-6 BCP-ALL cell line was used for a series of functional investigations to understand the function of Aiolos in the pathogenesis of BCP-ALL. The results demonstrated that lentivirus-mediated overexpression of Aiolos suppressed cell apoptosis and arrested the cell cycle at the G0/G1 phase, which possibly contributed to growth inhibition of the Nalm-6 cells. Furthermore, these changes were possibly associated with downregulation of PTEN and phosphorylation of Akt.

At least 16 different isoforms of Aiolos exist, resulting from alternate splicing, have different cellular localisations and have the ability to alter the localisation of other Ikaros members (13). However, the AIO1 transcript comprises $>80 \%$ of the Aiolos isoforms in B-cells (14). To mimic the isoforms and cellular localizations of Aiolos in B-cells, the pWPT-PURO-GFP-Aiolos plasmid, containing the entire Aiolos coding sequence, was constructed and lentivirus-mediated transduction of Nalm-6 cells was performed to create a stable transfection cell line. The expression of the Aiolos isoform has been previously investigated in lymphoid development, however, its expression in unaffected Nalm- 6 cells remains to be elucidated. In the present study, untransfected Nalm- 6 cells expressed low levels of Aiolos at the RNA and protein levels. The results indicated that Nalm-6 cells were successfully transduced with the lentivirus and that Aiolos was successfully overexpressed in the Nalm- 6 cells.

Aiolos is undoubtedly important in the control of mature B-lymphocyte differentiation and proliferation (15). Aiolos 

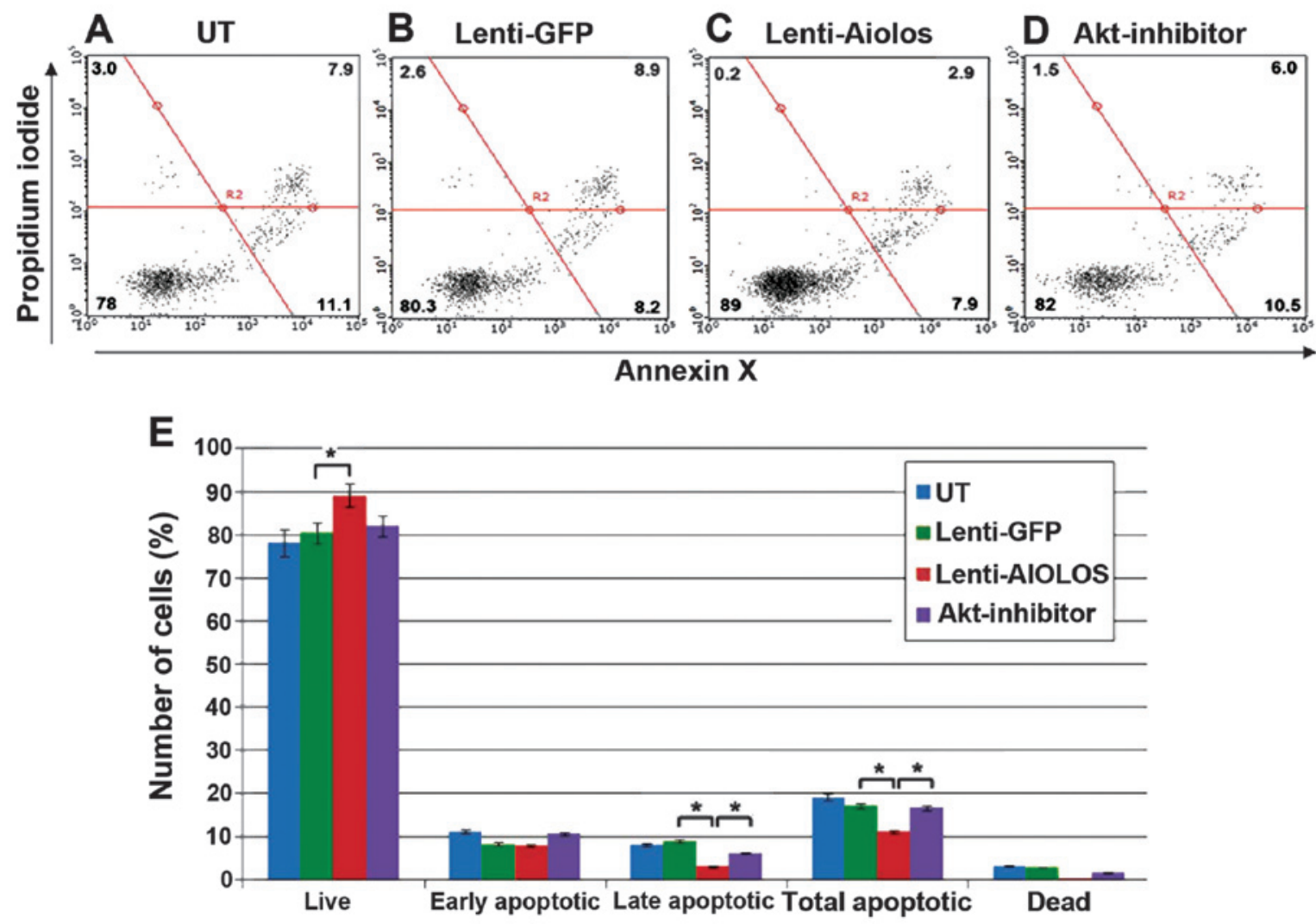

Figure 3. Effect of overexpression of Aiolos on Nalm-6 cell apoptosis. (A-C) Flow cytometric analysis demonstrated that the proportion of total apoptotic cells decreased in the Nalm-6 cells with increased expression of aiolos. (D and E) Total number of apoptotic cells decreased in Aiolos-transfected Nalm-6 cells compared with those in the Lenti-GFP group ("P<0.05). The difference between the Aiolos-transfected and the UT Nalm- 6 cells in the percentage of early apoptotic cells was marginal, whereas the difference in the percentage of late apoptotic cells was significant $\left({ }^{*} \mathrm{P}<0.05\right)$. Akti- $1 / 2$ effectively increased the percentage of apoptotic cells in the Aiolos-transfected Nalm- 6 cells (" $\mathrm{P}<0.05$ vs. Aiolos-transfected group). Error bars indicate the standard deviation of the mean of three experiments. GFP, green fluorescent protein; UT, untransfected control; Lenti-GFP, lentiviral vector control; Lenti-Aiolos, Aiolos-transfected.
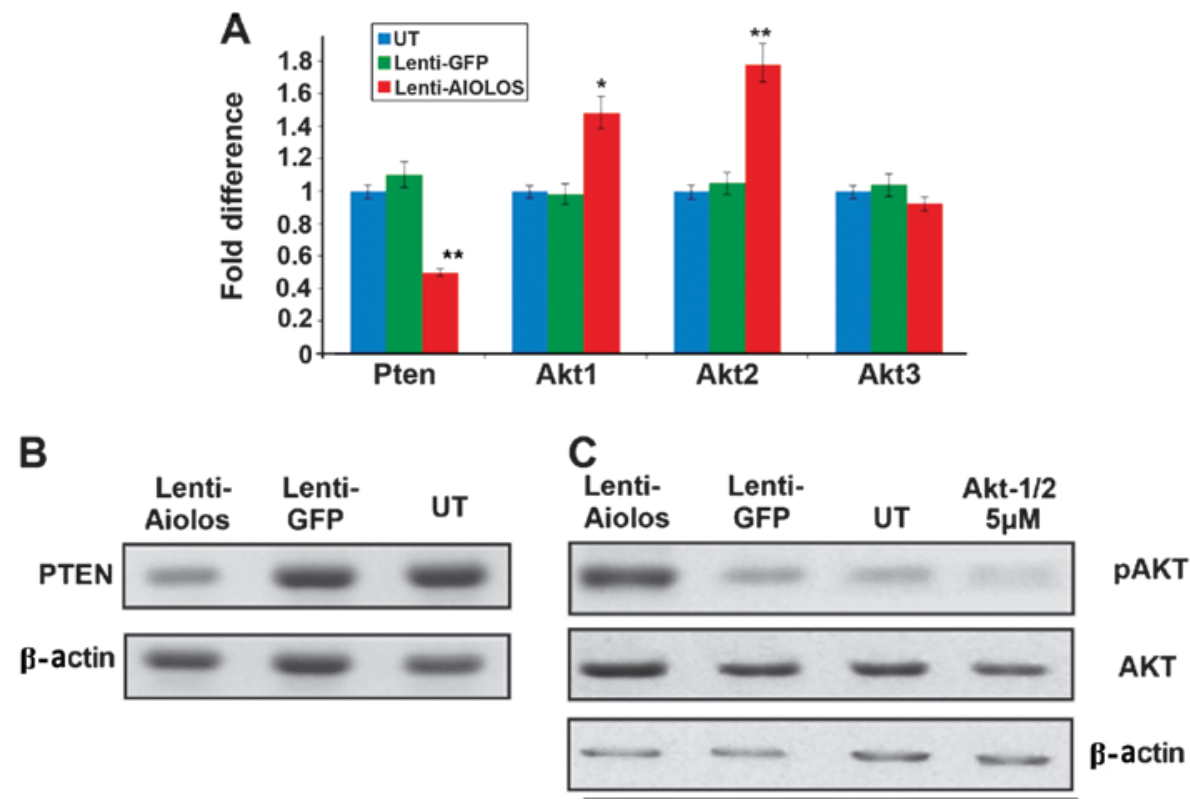

Figure 4. Upregulated Aiolos suppresses the expression of PTEN, but activated certain PI3K/Akt signalling pathway-associated genes in the Nalm-6 cells. (A) Reverse transcription-quantitative polymerase chain reaction revealed that the relative transcript levels of PTEN were markedly reduced in the Nalm-6 cells of the Lenti-Aiolos group compared with those in the Lenti-GFP group $\left({ }^{* *} \mathrm{P}<0.01\right)$. The upregulation of Akt1 ("P<0.05) and Akt2 $\left({ }^{(* *} \mathrm{P}<0.01\right)$ was confirmed in the Lenti-Aiolos group compared with the control group. No significant change was observed in the expression of Akt3. $\beta$-actin was used as an internal control. Error bars indicate the standard deviation of the mean from three experiments. (B) Western blotting results revealed the expression of PTEN decreased in the Nalm-6 cells of Lenti-Aiolos group and that the (C) protein expression of Akt did not change considerably, whereas the levels of pAkt were significantly higher in the Lenti-Aiolos group compared with the control group. Treatment with $5 \mu \mathrm{M}$ Akti-1/2 for $48 \mathrm{~h}$ effectively inhibited the phosphorylation of Akt. $\beta$-actin served as a loading control. PTEN, phosphatase and tensin homolog deleted on chromosome ten; PI3K, phosphatidylinositol-3-kinase; GFP, green fluorescent protein; pAkt, phosphorylated Akt; Lenti-GFP, lentiviral vector control; Lenti-Aiolos, Aiolos-transfected. 
silences the expression of the surrogate light-chain gene $\lambda 5$, thereby downregulating the pre-B-cell receptor (pre-BCR) (16). In addition, Aiolos directly binds to the c-Myc promoter and represses the expression of c-Myc in pre-B cells. Repression of c-Myc by Aiolos subsequently leads to the expression of p27 and the downregulation of cyclin D3 (17). P27 is a type of cyclin-dependent kinase inhibitor protein, which inhibits G1/S conversion and negatively regulates the cell cycle by inhibiting the functions of cell cycle regulators, including cyclin D3 $(18,19)$. By contrast, pre-BCR, cyclin D3 and c-Myc are necessary for the proliferation of pre-B-cells (20-22). Consistent with results of previous studies, the present study demonstrated that overexpression of Aiolos suppressed proliferation of Nalm- 6 cells. In addition, the results of cell cycle analysis indicated that upregulation of Aiolos arrested the Nalm- 6 cells at the G0/G1 phase.

Previous tudies have confirmed that overexpression of Aiolos in Nalm-6 cells inhibits cell apoptosis, which is consistent with previous studies demonstrating that disruption of Aiolos accelerates premature B cell apoptosis, mediated by BCR signalling through elevation in the release of cytochrome $c(23,24)$. However, different findings have been reported regarding the function of cyclin D3 in the apoptotic process of B cells. Wang et al (25) reported that downregulation of cyclin D3 increases the number of B-cell CLL cells undergoing apoptosis and another study suggested that repression of c-Myc and cyclin D3 is necessary to arrest human leukaemia cells at the G1 phase of the cell cycle, but neither is required for apoptosis (26). Therefore, in addition to c-Myc and cyclin D3, Aiolos potentially acts through other mechanisms to regulate the apoptotic process.

The present results demonstrate the crucial function of PTEN. The expression of PTEN was significantly reduced in Aiolos-overexpressed Nalm-6 cells. Previous studies have identified the functions of PTEN in leukaemogenesis (27-29), however, the functions of PTEN in association with Aiolos remain to be elucidated.PTEN was initially identified as a tumour suppressor, and loss or mutation of PTEN is reported to be associated with the development of various types of cancer $(30,31)$. PTEN may exert this regulatory role by altering its own expression and activity in response to external stimuli, including cigarette smoking or cytokines (32-35). PTEN primarily acts downstream of various other pathways as a negative regulator of the PI3K pathway due to its lipid phosphatase activity. Loss of PTEN increases the level of PI3-phosphate, thereby mimicking the effect of constitutive PI3K activation (36-38). Accumulation of PI3-phosphate activates various protein kinases, including Akt kinases (39). In addition to the reduction in PTEN levels, the results of the present study demonstrated that the expression and phosphorylation of Akt increased. This was consistent with the findings of the previously mentioned studies (40). Akt is the central node in the PTEN-regulated pathway, and activated Akt can act on multiple downstream targets, which are involved in proliferation, cell metabolism and apoptosis (40). Thus, improved cell survival may be attributed to the activated Akt pathway in the Aiolos-overexpressed Nalm-6 cells.

Previous studies have demonstrated that, in addition to inhibiting apoptosis, activated Akt can promotes cell cycle progression and cell growth (41-43). However, the present study revealed that overexpression of Aiolos inhibited the growth of Nalm- 6 cells and arrested the cells at the G0/G1 phase. Thus, the loss of PTEN may be insufficient to provide a proliferative advantage in BCP-ALL cells. Downregulation of c-Myc and cyclin D3 was sufficient for growth inhibition in the Aiolos-overexpressed Nalm-6 cells, despite proliferation signals, driven by the loss of PTEN. Subsequently, chemical inhibitors of Akt (Akti-1/2) were used to verify whether Akt is a key regulatory factor of apoptosis or growth regulation. The findings indicated that inhibiting the phosphorylation of Akt promoted the apoptosis of Aiolos-overexpressed Nalm- 6 cells. Akti-1/2 inhibited Akt, however, it did not induce changes in proliferation or the cell cycle. The results further demonstrated that overexpression of Aiolos induced changes in PTEN, and that Akt affected BCP-ALL cell apoptosis. Certain previous studies have indicated that loss of PTEN, which positively regulates Akt signalling, leads to the expansion of leukaemic stem cell subpopulations (44). However, loss of PTEN does not exert a positive effect in promoting a short-term increase in the total number of cells.

In conclusion, the results of the present study indicated that upregulation of the expression of Aiolos in Nalm- 6 cells inhibited cell proliferation, suppressed apoptosis and arrested the cell cycle at the G0/G1 phase. These findings revealed two critical functions of Aiolos in Nalm- 6 cells; to limit pre-B-cell expansion via c-Myc and cyclin D3-dependent pathways and to improve pre-B cell survival via PTEN- and Akt-dependent processes. Characterising these potential genetic interactions may provide a foundation for further investigation of the pathogenesis of BCP-ALL.

\section{Acknowledgements}

The present study was supported by the National Natural Science Foundation of China (no. 81102710), the Science and Technology Plan of Shandong Province (nos. 2009GG10002041 and 2009GG2HZ02003) and the Independent Innovation Foundation of Shandong University (no. 2012TS149).

\section{References}

1. Kaatsch P: Epidemiology of childhood cancer. Cancer Treat Rev 36: 277-285, 2010.

2. Pui CH, Robison LL and Look AT: Acute lymphoblastic leukaemia. Lancet 371: 1030-1043, 2008.

3. Cobaleda C and Sánchez-Garcia I: B-cell acute lymphoblastic. leukaemia: towards understanding its cellular origin. Bioessays 31 : 600-609, 2009.

4. Georgopoulos K, Moore DD and Derfler B: Ikaros, an early lymphoid-specific transcription factor and a putative mediator for T cell commitment. Science 258: 808-812, 1992.

5. Kelley CM, Ikeda T, Koipally J, et al: Helios, a novel dimerization partner of Ikaros expressed in the earliest hematopoietic progenitors. Curr Biol 8: 508-515, 1998.

6. Morgan B, Sun L, Avitahl N, et al: Aiolos, a lymphoid restricted transcription factor that interacts with Ikaros to regulate lymphocyte differentiation. EMBO J 16: 2004-2013, 1997.

7. Georgopoulos K, Winandy S and Avitahl N: The role of the Ikaros gene in lymphocyte development and homeostasis. Annu Rev Immunol 15: 155-176, 1997.

8. Wang JH, Avitahl NA, Cariappa C, et al: Aiolos regulates B cell activation and maturation to effector state. Immunity 9: 543-553, 1998.

9. Nakase K, Ishimaru F, Avitahl N, et al: Dominant negative isoform of the Ikaros gene in patients with adult B-cell acute lymphoblastic leukemia. Cancer Res 60: 4062-4065, 2000.

10. Nuckel H, Frey UH, Sellmann L, et al: The IKZF3 (Aiolos) transcription factor is highly upregulated and inversely correlated with clinical progression in chronic lymphocytic leukaemia. $\mathrm{Br}$ J Haematol 144: 268-270, 2009. 
11. Billot K, Soeur J, Chereau F, et al: Deregulation of Aiolos expression in chronic lymphocytic leukemia is associated with epigenetic modifications. Blood 117: 1917-1927, 2011.

12. Antica M, Cicin-Sain L, Kapitanovic S, et al: Aberrant Ikaros, Aiolos and Helios expression in Hodgkin and non-Hodgkin lymphoma. Blood 111: 3296-3297, 2008.

13. Caballero R, Setien F, Lopez-Serra L, et al: Combinatorial effects of splice variants modulate function of Aiolos. J Cell Sci 120 2619-2630, 2007.

14. Duhamel M, Arrouss I, Merle-Béral H, et al: The Aiolos transcription factor is up-regulated in chronic lymphocytic leukemia. Blood 111: 3225-3228, 2008.

15. Cobb BS and Smale ST: Ikaros-family proteins: in search of molecular functions during lymphocyte development. Curr Top Microbiol Immunol 290: 29-47, 2005.

16. Thompson EC, Cobb BS, Sabbattini P, et al: Ikaros DNA-binding proteins as integral components of B-cell developmental-stage-specific regulatory circuits. Immunity 26 : 335-344, 2007.

17. Ma S, Pathak S, Mandal M, et al: Ikaros and Aiolos inhibit pre-B-cell proliferation by directly suppressing c-Myc expression. Mol Cell Biol 30: 4149-4158, 2010.

18. Myung DS, Park YL, Chung CY, et al: Expression of livin in colorectal cancer and its relationship to tumor cell behavior and prognosis. PLoS One 8: e73262, 2013.

19. Toyoshima H and Hunter T: P27, a novel inhibitor of G1 cyclin-Cdk protein kinase activity, is related to p21. Cell 78: 67-74, 1994.

20. Herzog S, Reth M and Jumaa H: Regulation of B-cell proliferation and differentiation by pre-B-cell receptor signaling. Nat Rev Immunol 9: 195-205, 2009.

21. Cooper AB, Sawai CM, Sicinska E, et al: A unique function for cyclin D3 in early B cell development. Nat Immunol 7 : 489-497, 2006

22. Habib T, Park H, Tsang M, et al: Myc stimulates B lymphocyte differentiation and amplifies calcium signaling. J Cell Biol 179: 717-731, 2007.

23. Narvi E, Nera KP, Terho P, et al: Aiolos controls gene conversion and cell death in DT40 B cells. Scand J Immunol 65: 503-513, 2007.

24. Kikuchi H, Yamashita K, Nakayama M, et al: Lacking of Aiolos accelerates pre-mature B cell apoptosis mediated by BCR signaling through elevation in cytochrome c release. Biochim Biophys Acta 1793: 1304-1314, 2009.

25. Wang P, Pavletic ZS and Joshi SS: Increased apoptosis in B-chronic lymphocytic leukemia cells as a result of cyclin D3 down regulation. Leuk Lymphoma 43: 1827-1835, 2002.

26. Ausserlechner MJ, Obexer P, Böck G, et al: Cyclin D3 and c-MYC control glucocorticoid-induced cell cycle arrest but not apoptosis in lymphoblastic leukemia cells. Cell Death Differ 11: 165-174, 2004.

27. Shehata M, Schnabl S, Demirtas D, et al: Reconstitution of PTEN activity by CK2 inhibitors and interference with the PI3-K/Akt cascade counteract the antiapoptotic effect of human stromal cells in chronic lymphocytic leukemia. Blood 116: 2513-2521, 2010.

28. Zhiyong C, Wentong L, Xiaoyang Y, et al: PTEN Regulates VEGF, VEGFR1 Expression and Its Clinical Significance in Myeloid Leukemia. Blood (ASH Annual Meeting Abstracts) 114: Abstract 1001, 2009.
29. Gutierrez A, Sanda T, Grebliunaite R, et al: High frequency of PTEN, PI3 K and AKT abnormalities in T-cell acute lymphoblastic leukemia. Blood 114: 647-650, 2009.

30. Karoui M, Tresallet C, Julie C, et al: Loss of heterozygosity on $10 \mathrm{q}$ and mutational status of PTEN and BM PR1A in colorectal primary tumours and metastases. Br J Cancer 90: 1230-1234, 2004.

31. Poetsch M, Lorenz G and Kleist B: Detection of new PTEN/MM AC 1 mutations in head and neck squamous cell carcinomas with loss of chromosome 10. Cancer Genet Cytogenet 132: 20-24, 2002

32. Hou R, Zhang J, Yin T, et al: Upregulation of PTEN by peroxynitrite contributes to cytokine-induced apoptosis in pancreatic beta-cells. Apoptosis 15: 877-886, 2010.

33. Koul D, Yao Y, Abbruzzese JL, et al: Tumor suppressor MMAC/PTEN inhibits cytokine-induced NFkappa B activation without interfering with the IkappaB degradation pathway. J Biol Chem 276: 11402-11408, 2001.

34. Lee SY, Kang EJ, Hur GY, et al: Peroxisome proliferator-activated receptor-gamma inhibits cigarette smoke solution-induced mucin production in human airway epithelial (NCI-H292) cells. Am J Physiol Lung Cell Mol Physiol 291: L84-L90, 2006.

35. Kim S, Domon-Dell C, Kang J, et al: Down-regulation of the tumor suppressor PTEN by the tumor necrosis factor-alpha/nuclear factor-kappaB (NF-kappaB)-inducing kinase/NF-kappaB pathway is linked to a default IkappaB-alpha autoregulatory loop. J Biol Chem 279: 4285-4291, 2004.

36. Maehama $\mathrm{T}$ and Dixon JE: The tumor suppressor, PTEN/MMAC1, dephosphorylates the lipid second messenger, phosphatidylinositol 3,4,5-trisphosphate. J Biol Chem 273: 13375-13378, 1998.

37. Stambolic V, Suzuki A, de la Pompa JL, et al: Negative regulation of PKB/Akt-dependent cell survival by the tumor suppressor PTEN. Cell 95: 29-39, 1998.

38. Sun H, Lesche R, Li DM, et al: PTEN modulates cell cycle progression and cell survival by regulating phosphatidylinositol 3,4,5,-trisphosphate and Akt/protein kinase B signaling pathway. Proc Natl Acad Sci USA 96: 6199-6204, 1999.

39. Gold MR, Scheid MP, Santos L, et al: The B cell antigen receptor activates the Akt (protein kinase B)/glycogen synthase kinase-3 signaling pathway via phosphatidylinositol 3-kinase. J Immunol 163: 1894-1905, 1999.

40. Leslie NR and Downes CP: PTEN function: how normal cells control it and tumour cells lose it. Biochem J 382: 1-11, 2004

41. Datta SR, Dudek H, Tao X, et al: Akt phosphorylation of BAD couples survival signals to the cell-intrinsic death machinery. Cell 91: 231-241, 1997.

42. Salmena L, Carracedo A and Pandolfi PP: Tenets of PTEN tumor suppression. Cell 133: 403-414, 2008.

43. Perry JM, He XC, Sugimura R, et al: Cooperation between both Wnt/\{beta\}-catenin and PTEN/PI3 K/Akt signaling promotes primitive hematopoietic stem cell self-renewal and expansion. Genes Dev 25: 1928-1942, 2011.

44. Tothova $\mathrm{Z}$ and Gilliland DG: FoxO transcription factors and stem cell homeostasis: Insights from the hematopoietic system. Cell Stem Cell 16: 140-152, 2007. 